\title{
ON THE DIMENSION OF PRODUCT SPACES AND AN EXAMPLE OF M. WAGE
}

\author{
TEODOR C. PRZYMUSIŃSKI ${ }^{1}$
}

\begin{abstract}
Modifying a recent example obtained under the assumption of the Continuum Hypothesis by Michael Wage, we prove, without any settheoretic assumptions beyond ZFC, that for every natural number $n$ there exists a separable and first countable space $X$ such that:

(a) $X^{n}$ is Lindelö and $\operatorname{dim} X^{n}=0$;

(b) $X^{n+1}$ is normal but $\operatorname{dim} X^{n+1}>0$.

We obtain from this the following corollary. There exists a separable and first countable Lindelof space $X$ such that:

(a) $\operatorname{dim} X=0$;

(b) $X^{2}$ is normal but $\operatorname{dim} X^{2}>0$.

The space $X$ instead of being Lindelö can be made locally compact and locally countable.
\end{abstract}

Assuming the Continuum Hypothesis $(\mathrm{CH})$ Michael Wage constructed a beautiful example of a strongly zerodimensional space $X$ such that $X^{2}$ is not strongly zerodimensional, thus giving a consistent negative answer to a long outstanding problem in dimension theory, namely, whether $\operatorname{dim} X \times Y<$ $\operatorname{dim} X+\operatorname{dim} Y[\mathbf{W}]$.

In this paper we modify his construction and prove, without any set-theoretic assumptions beyond $\mathrm{ZFC}$, the following:

THEOREM. For every natural number $n$ there exists a separable and first countable space $X$ such that:

(a) $X^{n}$ is Lindelöf and $\operatorname{dim} X^{n}=0$;

(b) $X^{n+1}$ is normal but $\operatorname{dim} X^{n+1}>0$.

COROLlary. There exists a separable and first countable Lindelöf space $X$ such that:

(a) $\operatorname{dim} X=0$;

(b) $X^{2}$ is normal but $\operatorname{dim} X^{2}>0$.

Thus our theorem provides a "real" counterexample to the conjecture that $\operatorname{dim} X \times Y \leqslant \operatorname{dim} X+\operatorname{dim} Y$ in the class of normal spaces. The space $X$,

Received by the editors October 1, 1978.

AMS (MOS) subject classifications (1970). Primary 54F45, 54B10, 54D20.

Key words and phrases. Strongly zerodimensional spaces, product spaces, normality, Lindelöf property.

${ }^{1}$ The result presented in this paper was obtained while the author was a Visiting Assistant Professor at the University of Pittsburgh in 1976/77. 
instead of being Lindelöf, can be made locally compact and locally countable.

The author wishes to point out that the basic idea used in the proof of our theorem is the same as in [W] and belongs to M. Wage. We avoid the assumption of $\mathrm{CH}$ by: (1) replacing Kunen's technique originally used by $\mathbf{M}$. Wage by a technique invented by van Douwen [vD], and (2) an appropriate application of methods developed by the author in $[\mathrm{P}]$ which involve the notion of $n$-cardinality $\left[\mathbf{P}_{1}\right]$. These methods are also crucial to ensure the normality of $X^{2}$ (or $X^{n+1}$ ) and the Lindeloffness of $X^{n}$. (We note that the method suggested by M. Wage in [W] to make the square $X^{2}$ of his space $X$ normal was incorrect.)

Let us recall that for a nonempty normal space $X: \operatorname{dim} X=0$ iff Ind $X=$ 0 iff any two disjoint closed subsets of $X$ can be separated by a closed-andopen subset of $X$. Spaces having this property are also called strongly zerodimensional. Every zerodimensional Lindelöf space is strongly zerodimensional (cf. [E]).

A real-valued function $f: X \rightarrow R$ is upper (resp. lower) semicontinuous if for every $r \in R$ the set $f^{-1}((-\infty, r))\left(\right.$ resp., $\left.f^{-1}((r, \infty))\right)$ is open in $X$. A function which is either upper or lower semicontinuous is called semicontinuous.

For a real-valued function $g: I^{m} \rightarrow R$, where $I$ is the unit interval, by a $g$-topology on $I^{m}$ we shall understand a topology generated by the metric $d$ defined by $d(x, y)=|x-y|+|g(x)-g(y)|$, for $x, y \in I^{m}$. Clearly the $g$-topology is finer than the natural topology on $I^{m}$. Sets open in the $g$-topology will be called $g$-open, those closed in the $g$-topology $g$-closed, etc. By $\Delta \subset I^{m}$ we shall denote the diagonal in $I^{m}$ and we shall often identify $I$ with $\Delta$ using the natural correspondence $x \leftrightarrow(x, x, \ldots)$. By $\bigoplus_{i=1}^{m} X_{i}$ we denote the topological sum of spaces $X_{i}$.

$M$. Wage proved $[\mathbf{W}]$ the following lemma.

LEMMA 1. There exists an upper semicontinuous function $f: I \rightarrow I$ such that

(1) $f^{-1}(1)$ is uncountable;

(2) every nonempty $f$-open subset of I disjoint from $f^{-1}(0)$ has an uncountable f-boundary.

Our theorem is an easy consequence of Lemma 1 and the following lemma which seems to be interesting in itself.

LEMMA 2. For every natural number $n$ and every semicontinuous function $f$ : $I \rightarrow I$ there exist topologies $\tau_{1}, \tau_{2}, \ldots, \tau_{n+1}$ on I finer than the natural topology on $I$ and such that the space $X=\bigoplus_{i=1}^{n+1}\left(I, \tau_{i}\right)$ satisfies the following three conditions:

(3) $X^{n}$ is first countable, separable, strongly zerodimensional and Lindelöf;

(4) $X^{n+1}$ is normal and nomparacompact;

(5) the topology $\tau=\prod_{i=1}^{n+1} \tau_{i}$ on $I^{n+1}$ restricted to $\Delta \subset I^{n+1}$ is finer than the 
f-topology on $\Delta$, but at the same time for any $A, B \subset \Delta$, if $\left|\bar{A}^{f} \cap \bar{B}^{f}\right|>\omega$, then $\left|\bar{A}^{\tau} \cap \bar{B}^{\tau}\right|=2^{\omega}=c$.

Let us notice that for an arbitrary continuous mapping $f: I \rightarrow I$ Lemma 2 immediately yields a Lindelöf space $X$ such that $X^{2}$ is normal but not paracompact. The first "real" example of such a space was obtained in [P]. Basically, Lemma 2 says that for a given semicontinuous function $f$ there exist "decent" topologies $\tau_{i}$ on $I$ such that the subspace topology induced on $\Delta$ by the product topology $\tau$ on $I^{n+1}$ is very "close" to the $f$-topology on $\Delta$ (thus, if the $f$-topology is "bad" then so may be $\tau$ ).

Let us first derive our theorem from Lemmas 1 and 2. Take an arbitrary $n, f$ as described in Lemma 1 and the space $X$ guaranteed by Lemma 2. It suffices to verify that $\operatorname{dim} X^{n+1}>0$ and to this end it is enough to show that the subspace $\Delta$ of $\left(I^{n+1}, \tau\right)$ is not strongly zerodimensional. We shall show (cf. [W]) that closed subsets $f^{-1}(0)$ and $f^{-1}(1)$ of $\Delta$ cannot be separated by a closed-and-open subset of $(\Delta, \tau)$.

Suppose that $U$ is a closed-and-open subset of $(\Delta, \tau), f^{-1}(0) \subset U$ and $f^{-1}(1) \cap U=\varnothing$. Since $\bar{U}^{\tau} \cap \overline{\Delta \backslash U^{r}}=\varnothing$, by (5) the set $F=\bar{U}^{f} \cap \overline{\Delta \backslash U^{f}}$ is countable. Thus by (1) the f-open set $W=\Delta \backslash \bar{U}^{f} \supset \Delta \backslash(U \cup F)$ is nonempty. By (2) $\mathrm{Fr}_{f} W$ is uncountable, but $\mathrm{Fr}_{f} W \subset F$, which is a contradiction.

Proof of LemMa 2. Let $f: I \rightarrow I$ be a semicontinuous function on $I$. Without loss of generality we can assume that $f$ is upper semicontinuous. In order to simplify the construction we shall also assume that $n=1$; the proof of the general case is completely analogous.

By a result of Baire (see [E, Problem 1.7.15 (c)]) there exists a sequence $\left\{f_{1 / n}\right\}_{n=1}^{\infty}$ of continuous real-valued functions $f_{1 / n}: I \rightarrow R$ such that:

(6) $f(x)<f_{1 /(n+1)}(x) \leqslant f_{1 / n}(x)$, for $x \in I$ and $n=1,2, \ldots$,

(7) $\lim _{1 / n \rightarrow 0} f_{1 / n}(x)=f(x)$, for every $x \in I$.

Using functions $f_{1 / n}$ one easily defines a family $\left\{f_{t}\right\}_{t \in(0,1]}$ of continuous real-valued functions $f_{t}: I \rightarrow R$ satisfying the following three conditions (just define $f_{t}$ as a suitable linear combination of functions $f_{1 / n}$ and $f_{1 /(n+1)}$, if $1 /(n+1)<t<1 / n)$ :

(8) $f(x)<f_{t}(x) \leqslant f_{s}(x)$, for $t \leqslant s$ and $x \in I$;

(9) $\lim _{t \rightarrow 0} f_{t}(x)=f(x)$, for every $x \in I$;

(10) the family $\left\{f_{t}\right\}_{t \in(0,1]}$ is continuous with respect to $t$, i.e., $f_{t_{0}}=\lim _{t \rightarrow t_{0}} f_{t}$ (pointwise or-equivalently-uniform), for $t_{0} \in(0,1]$.

Let us define a function $g: I^{2} \rightarrow R$ by the following formula:

$$
g(x, y)= \begin{cases}f(x), & \text { if } x=y \\ \max \left(f_{|x-y|}(x), f_{|x-y|}(y)\right), & \text { if } x \neq y\end{cases}
$$

One easily checks that $g$ has the following properties:

(11) $g(x, x)=f(x)$;

(12) $g$ is upper semicontinuous; 
(13) for any open $V \subset R$ the set $g^{-1}(V) \backslash \Delta$ is open in $I^{2}$ (in particular, $g \mid I^{2} \backslash \Delta$ is continuous);

(14) for every $x \in I$ the open subset $G_{x}=g^{-1}((f(x), \infty)) \backslash \Delta$ of $I^{2}$ contains all points $(u, v) \in I^{2}$ such that $u \neq v$ and either $u=x$ or $v=x$.

By (11) the $g$-topology on $\Delta$ coincides with the $f$-topology on $\Delta$; by (13) the $g$-topology on $I^{2} \backslash \Delta$ coincides with the natural topology on $I^{2} \backslash \Delta$ and (12) implies

(15) for every $(x, x) \in \Delta$ and its $g$-neighbourhood $W$ there exists an open neighbourhood $U$ of $(x, x)$ in $I^{2}$ such that $U \cap G_{x} \subset W$.

Indeed, there exist $r, s \in R$ such that $r<f(x)<s$ and an open neighbourhood $V$ of $(x, x)$ in $I^{2}$ such that $V \cap g^{-1}((r, s)) \subset W$. For $U=V$ $\cap g^{-1}((-\infty, s))$ we have

$$
U \cap G_{x} \subset V \cap g^{-1}((-\infty, s)) \cap g^{-1}((r,+\infty))=V \cap g^{-1}((r, s)) \subset W .
$$

Let us recall that the 2-cardinality $\left[\mathbf{P}_{1}\right]$ of a subset $A \subset I^{2}$ is defined by

(16) $|A|_{2}=\max \{|B|: B \subset A$ and $\hat{x} \cap \hat{y}=\varnothing$, for $x, y \in B, x \neq y\}$, where $\hat{x}=\left\{x_{1}, x_{2}\right\} \subset I$, for $x=\left(x_{1}, x_{2}\right) \in I^{2}$, and that

(17) $|A|_{2}=\min \{|S|: S \subset I$ and $A \subset S \times I \cup I \times S\}$, provided that $|A|_{2}$ is infinite.

Since $I$ with the $f$-topology is homeomorphic to a Borel subspace of $I^{2}$ (the graph of $f$ ) every $f$-closed uncountable subset of $I$ has cardinality continuum (see [Ku, §31, VII, Theorem 1 and §37, I, Theorem 1]). ${ }^{2}$ This, together with (13) and Theorem 1 from $\left[\mathbf{P}_{\mathbf{1}}\right]$, easily implies that

(18) every 2-uncountable $g$-closed subset of $I^{2}$ has 2-cardinality continuum.

Arguing as in the proof of Theorem 2 from $\left[\mathbf{P}_{\mathbf{1}}\right]$ one proves that there exist disjoint subsets $C_{0}, C_{1}, C_{2}$ of $I$ such that $I=C_{0} \cup C_{1} \cup C_{2}$ and

(19) $\left|C_{j}^{2} \cap F\right|_{2}=c$, for every $j=0,1,2$ and every 2 -uncountable $g$-closed subset $F$ of $I^{2}$.

Let $\prec$ be an arbitrary well-ordering of $I$ of type $c$. For $i=1,2$ we shall construct a topology $\tau_{i}$ on $I$ so that the following five conditions are satisfied:

(20) points from $C_{i}$ have a local base consisting of open intervals (with respect to the usual ordering of $I$ );

(21) every point has a countable local base consisting of closed subsets of $I$;

(22) the topology $\tau=\tau_{1} \times \tau_{2}$ on $I^{2}$ is finer than the $g$-topology on $I^{2}$;

(23) for any $A, B \subset I^{2}$, if $\left|\bar{A}^{g} \cap \bar{B}^{g}\right|_{2}>\omega$, then $\left|\bar{A}^{\tau} \cap \overline{B^{\tau}}\right|_{2}=c$;

(24) for every $x \in I$ the set $\{(y, y) \in \Delta: y \preccurlyeq x\}$ is open in $(\Delta, \tau)$.

Let us first show that topologies $\tau_{i}$ fulfill the requirements of Lemma 2, for $n=1$. Since the $g$-topology is finer than the natural topology on $I^{2}$, condition (22) implies that topologies $\tau_{i}$ are finer than the natural topology on $I$. Let $X_{i}=\left(I, \tau_{i}\right)$ and $X=X_{1} \oplus X_{2}$. By (21) the space $X$ is first countable and zerodimensional, hence regular. To see that $X$ is Lindelöf it suffices to use (20) and observe that, by (19), for every $i=1,2$ the complement $I \backslash U$ of any

${ }^{2}$ This was pointed out by $R$. Pol. 
open subset $U$ of $I$ containing $C_{i}$ is countable. Since $X$ is Lindelöf and zerodimensional it is strongly zerodimensional.

The normality of $X^{2}$ follows from (22), (23) and (20). It suffices to show that for every $i, j=1,2$ the space $Y=X_{i} \times X_{j}$ is normal. If $i=j$ then by (20) $Y$ is Lindelö, because in virtue of (19) the complement of any open subset $U$ of $I^{2}$ containing $C_{i}^{2}$ is 2-countable and thus Lindelöf. To show that the space $Z=X_{1} \times X_{2}$ is normal, choose disjoint closed subsets $A, B$ of $Z$. By (23) the $g$-closed set $F=\bar{A}^{g} \cap \bar{B}^{g}$ is 2-countable and thus Lindelöf. Using this and (22) it is easy to construct an open covering $\left\{U_{m}\right\}_{m=1}^{\infty}$ of $Z$ such that for every $m$ either $\bar{U}_{m}^{\tau} \cap A=\varnothing$ or $\bar{U}_{m}^{\tau} \cap B=\varnothing$, which proves the normality of $Z$ and of $X^{2}$ (cf. [vD] or [P]).

The space $X$ is also $\omega_{1}$-compact. ${ }^{3}$ Indeed, let $A$ be an uncountable subset of $Z$ and $B$ a $g$-dense countable subset of $A$. By (23), since $\bar{B}^{g} \cap \bar{B}^{g}=\bar{B}^{g} \supset A$ is uncountable, the set $\bar{B}^{\tau}$ is uncountable, which shows that $A$ is not discrete (cf. [vD]). Thus $X^{2}$ is $\omega_{1}$-compact and as (24) implies that $X^{2}$ is not Lindelöf, it cannot be paracompact. Condition (5) of Lemma 2 follows immediately from (23) and (11).

In order to simplify the construction, topologies $\tau_{i}$ that we are about to define will not be separable, but this can be easily corrected by choosing a countable $f$-dense subset $D$ of $I$ and requiring that every open in $\tau_{i}$ neighbourhood of a point $x \in I$ contain a "tail" of an $f$-convergent sequence of points of $D$.

It remains to construct topologies $\tau_{i}$ on $I$ so that conditions (20)-(24) are satisfied. For every $x \in I$ let $I(x)=\{y \in I: y \preccurlyeq x\}$ and let $\left\{\left(A_{s}, B_{s}\right)\right\}_{s<c}$ be the collection of all pairs $\left(A_{s}, B_{s}\right)$ of countable subsets of $I^{2}$ such that $\left|\bar{A}_{s}^{g} \cap \bar{B}_{s}^{g}\right|_{2}>\omega$. We can assume that every such pair appears continuum many times in the sequence $\left\{\left(A_{s}, B_{s}\right)\right\}_{s<c}$. For every $s<c$ there exists a $q_{s} \in I$ such that $A_{s} \cup B_{s} \subset I\left(q_{s}\right)^{2}$. Using (18) and (19) one can construct (cf. [P]) a transfinite sequence $\{p(s)\}_{s<c}$ of points $p(s)=\left(p_{1}(s), p_{2}(s)\right)$ of $I^{2}$ such that:

(25) $p(s) \in \bar{A}_{s}^{g} \cap \bar{B}_{s}^{g}$ and $\hat{p}(s) \subset C_{0}$;

(26) $\hat{p}(s) \cap \hat{p}(t)=\varnothing$, if $s \neq t$;

(27) $\hat{p}(s) \cap I\left(q_{s}\right)=\varnothing$.

Let $d$ be the metric on $I^{2}$ generating the $g$-topology on $I^{2}$, i.e. $d(x, y)=\mid x$ $-y|+| g(x)-g(y) \mid$. By transfinite recursion on $I$ we shall define for every $x \in I$ and $i=1,2$ bases $\left\{B_{k}^{i}(x)\right\}_{k=1}^{\infty}$ of neighbourhoods of $x$ in $\tau_{i}$, so that the following conditions are satisfied:

(28) sets $B_{k}^{i}(x)$ are closed subsets of $I$ and $B_{k+1}^{i}(x) \subset B_{k}^{i}(x)$;

(29) for every $x \in I$ the $d$-diameters of sets $B_{k}(x)=B_{k}^{1}(x) \times B_{k}^{2}(x)$ approach zero when $k \rightarrow \infty$;

(30) $B_{1}(x) \cap \Delta \subset\{(y, y) \in \Delta: y \preccurlyeq x\}$, for every $x \in I$.

Suppose that $x \in I$ and that such bases of neighbourhoods have been

\footnotetext{
${ }^{3}$ That is, every discrete closed subset of $X$ is countable.
} 
constructed for $y \prec x$. We consider three different cases:

(a) $x \in C_{i}$ for some $i=1,2$ or $x \in C_{0}$ and $x=p_{i}(s)$, for some $s<c$, but $p_{1}(s) \neq p_{2}(s)$

(b) $x \in C_{0}$ and $x \neq p_{i}(s)$, for every $i=1,2$ and $s<c$;

(c) $x \in C_{0}$ and $x=p_{1}(s)=p_{2}(s)$, for some $s<c$.

ad (a). We define $B_{k}^{i}(x)$ to be an arbitrary closed interval of length $<1 / k$, containing $x$, with endpoints from $C_{3-i}$ and we make sure that $B_{k+1}^{i}(x) \subset$ $B_{k}^{i}(x)$. We also put $B_{k}^{3-i}(x)=\{x\}$, for every $k$.

ad (b). We define $B_{k}^{1}(x)=B_{k}^{2}(x)=\{x\}$, for every $k=1,2, \ldots$

ad (c). From (25) it follows that $(x, x) \in \bar{A}_{s}^{g} \cap \bar{B}_{s}^{g}$ and thus using (14) it is not difficult to construct a sequence $\left\{\left(x_{m}, y_{m}\right)\right\}_{m=1}^{\infty}$ of points of $I^{2}$ such that $d\left(\left(x_{m}, y_{m}\right),(x, x)\right)<1 / m,\left(x_{m}, y_{m}\right) \in A_{s}$, if $m$ is even, $\left(x_{m}, y_{m}\right) \in B_{s}$, if $m$ is odd and $\left(x_{m}, y_{n}\right) \in G_{x}$, if $m \neq n$. There exist open in $I$ neighbourhoods $U_{m}$ and $V_{m}$ of points $x_{m}$ and $y_{m}$, respectively, such that $U_{m} \times V_{n} \subset G_{x}$, if $m \neq n$ and $\left(U_{m} \times V_{m}\right) \cap \Delta=\varnothing$, if $x_{m} \neq y_{m}$.

By (27), for every $m$ we have $x_{m} \prec x$ and $y_{m} \prec x$ and therefore the inductive hypothesis, (29) and (13) imply that for every $m$ there exists a $k_{m}$ such that $x_{m} \in B_{k_{m}}^{1}\left(x_{m}\right) \subset U_{m}, y_{m} \in B_{k_{m}}^{2}\left(y_{m}\right) \subset V_{m}$ and the $d$-diameter of $B_{k_{m}}^{1}\left(x_{m}\right) \times B_{k_{m}}^{2}\left(y_{m}\right)$ is less than $1 / m$. We put

$$
B_{k}^{1}(x)=\{x\} \cup \bigcup_{m>k} B_{k_{m}}^{1}\left(x_{m}\right) \text { and } B_{k}^{2}(x)=\{x\} \cup \bigcup_{m>k} B_{k_{m}}^{2}\left(y_{m}\right) .
$$

One easily checks that the above described systems of neighbourhood bases are well-defined and that conditions (28)-(30) are satisfied (to prove (29) use (15)). Let us briefly verify that the topologies $\tau_{i}$ determined by these systems of bases fulfill the requirements of (20)-(24). Clearly (20) and (21) are satisfied; (24) is an immediate consequence of (30); (22) follows from (29) and (13); and (23) is implied by the fact that for every $s<c$ we have $p(s) \in \bar{A}_{s}^{\tau} \cap \bar{B}_{s}^{\tau}$, where $\tau=\tau_{1} \times \tau_{2}$. This completes the proof of Lemma 2 and of our theorem.

Comments. (1) It is known that for normal spaces $Z, \operatorname{dim} Z=0$ iff Ind $Z=0$, thus in our theorem we also have Ind $X^{n}=0$, but Ind $X^{n+1}>0$.

(2) After the result presented in this paper was obtained, M. Wage informed the author that he was able to construct a "real" example of a Lindelöf space $X$ and a separable metric space $Y$ such that $\operatorname{dim} X=\operatorname{dim} Y$ $=0$, but $\operatorname{dim} X \times Y>0$. From [K] it follows however that the product space $X \times Y$ cannot be normal.

(3) It is easy to verify that actually our space $X$ satisfies $\operatorname{dim} X^{2}=1$.

ADDED IN PROOF. M. B. Charalambous pointed out that the space $X^{2}$ from our Corollary can also serve as an example of an $N$-compact space which is not strongly zerodimensional. First example of such a space was obtained by S. Mrówka. 


\section{REFERENCES}

[vD] E. van Douwen, A technique for constructing honest locally compact submetrizable examples, preprint.

[E] R. Engelling, General topolozy, PWN, Warsaw, 1977.

[K] Y. Kodama, On subset theorems and the dimension of products, Amer. J. Math. 91 (1969), 486-497.

[Ku] K. Kuratowski, Topology, vol. I, rev. ed., Academic Press, New York, London; PWN, Warsaw, 1966.

[P] T. Prymusinski, Normality and paracompactness in finite and countable Cartesian products, Fund. Math. 105 (1979).

$\left[P_{1}\right] \ldots$, On the notion of $n$-cardinality, Proc. Amer. Math. Soc. 6 (1978), 333-338.

[W] M. Wage, The dimension of product spaces, preprint.

Institute of Mathematics, Poush Academy of Sciences, Śnadectach 8 Warsaw, 00-950 Poland 